\title{
Developing the Role of the Clinical Academic Nurse, Midwife and Allied Health Professional in Healthcare Organisations
}

\author{
*Joanne Cooper ${ }^{\mathrm{abc}}$, Kay Mitchell ${ }^{\mathrm{de}}$, Alison Richardson ${ }^{\mathrm{ef}}$, and Louise Bramley ${ }^{\mathrm{ag}}$ \\ ${ }^{a}$ Institute of Nursing and Midwifery Care Excellence, Nottingham University Hospitals NHS Trust, United \\ Kingdom; ${ }^{b}$ Nottingham Digestive Diseases Centre, Nottingham University and NIHR Nottingham \\ Biomedical Research Centre, Nottingham University Hospitals NHS Trust, United Kingdom; ${ }^{c}$ Coventry \\ University, United Kingdom; ' NIHR Southampton Biomedical Research Centre, University of \\ Southampton, United Kingdom; ${ }^{e}$ University Hospital Southampton NHS Foundation Trust, United

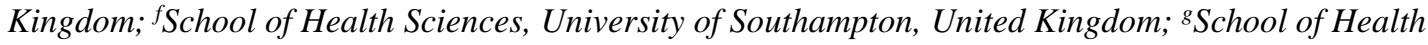 \\ Sciences, University of Nottingham, United Kingdom
}

\begin{abstract}
Clinical academics provide key contributions to positive outcomes in the delivery of highquality health and social care; however, building capacity and capability for these roles for Nurses, Midwives and Allied Health Professionals (NMAHPs) within contemporary healthcare settings is often complex and challenging. Accessing funding and training, such as that provided by the National Institute for Health Research (NIHR), can remain beyond the reach of NMAHPs at point-of-care delivery because of limited structural empowerment, practical support and a culture inhibiting the growth of clinical academic careers. This article will discuss strategic developments and partnerships from two organisations, both with a positive track record of supporting clinical academic career development for NMAHPs. We aim to provide practical and applicable examples showing how NMAHPs have been supported from foundational to post-doctoral level and outline these under three key headings: strategic commitment; structures to engage, enthuse and empower clinical academic careers; and realising the benefits for staff and patient experience. We contend that a wide-ranging level of support is required to encourage aspiring clinical academics to navigate this complex journey, often where the development of personal confidence, and access to early career models combining clinical and research activity are pivotal. We conclude that when crafted and created effectively with sustainable commitment by organisations, NMAHP clinical academics provide an innovative workforce solution with the knowledge and skills essential for a contemporary NHS healthcare system.
\end{abstract}

Keywords: clinical academic careers; National Health Service; integrated care systems; research

*Corresponding Author: Dr Joanne Cooper BSc, MA, PhD, RN (Adult). Assistant Director of Nursing and Midwifery, Institute of Nursing and Midwifery Care Excellence/Nottingham Digestive Diseases Centre and NIHR Nottingham Biomedical Research Centre at Nottingham University Hospitals NHS Trust, Derwent House, Flat 5, City Hospital Campus, Hucknall Road, Nottingham NG5 1PB, United Kingdom

Email: joanne.cooper3@nuh.nhs.uk

Journal URL: https://publications.coventry.ac.uk/index.php/pblh

Cooper, J., Mitchell, K., Richardson, A., \& Bramley, L. (2019). Developing the role of the clinical academic nurse, midwife and allied health professional in healthcare organisations. International Journal of Practice-based Learning in Health and Social Care, 7(2), 16-24. DOI 10.18552/ijpblhsc.v7i2.637

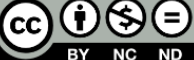

(C) BY ND 2019 Joanne Cooper, Kay Mitchell, Alison Richardson, and Louise Bramley. This Open Access article is distributed under the terms of the Creative Commons Attribution Attribution-Non-Commercial No Derivatives 4.0 International License (https://creativecommons.org/licenses/by-nc-nd/4.0/), which permits unrestricted noncommercial use, distribution, and reproduction in any medium, provided the original work is properly cited and is unaltered. 


\section{Introduction}

Research-active National Health Service (NHS) organisations in the United Kingdom are associated with improved outcomes in patient and staff experience compared to their less active counterparts (Jonker and Fisher 2018; Jonker et al., 2019). The introduction of research training programmes, such as the NIHR Integrated Clinical Academic Training (NIHR ICA) programme, provides NHS Trusts with vital funding on which to build a sustainable culture for research activity, specifically supporting clinical academic training for NMAHP professions.

The development of support structures and associated clinical academic roles within NHS Trusts is complex and can be limited by the contextual challenges faced by organisations and the difficulties in gaining access to sufficient funding across the career pathway. It is well recognised that contemporary healthcare and education must ensure that care provision and training is evidence-based and addresses the changing needs of patient populations. Experiences of authors of this article confirm the pivotal role of clinical academics in supporting excellence in care, development into careers of choice and improving patient outcomes through research and innovation methods.

The purpose of this article is to outline two exemplars, illustrating how NMAHPs have been supported to develop clinical academic roles, and their contribution to research and innovation in care. Examples range from foundational to post-doctoral level and are structured around the following headings: strategic commitment; structures to engage, enthuse and empower clinical academic careers; and realising the benefits for staff and patient experience.

\section{Strategic commitment}

Nottingham University Hospitals NHS Trust (NUH) is a large inner-city Trust. It provides services to over 2.5 million residents of Nottingham and its surrounding communities, and specialist services for a further 3-4 million people from across the region.

NUH is a research-active organisation, with an established research and innovation infrastructure, including an NIHR BRC and an active portfolio of clinical trial delivery (https://www.nuh.nhs.uk/nihrnottingham-biomedical-research-centre). However, while known for excellence in key areas of research led by staff within medicine, this was not reflected in opportunities, capacity or capability for aspiring clinical academic NMAHPs. A proactive response to this was instigated in 2011, with a specific focus on the nursing and midwifery professions.

In 2011, a collaborative funding model was agreed between NUH's Research and Innovation (R\&I) Department and Corporate Nursing in order to establish the Trust's first senior role responsible for building capacity and capability for Nursing and Midwifery (N\&M) clinical academic careers. This reflected a strategic executive commitment to building capacity across the professions. As a senior member of the N\&M and R\&I teams, the post-holder was able to raise the profile of N\&M Clinical Academic Careers (CACs), embedding aims for research and evidence-based practice within the N\&M strategy and further developing strategic leadership and partnerships across internal and external stakeholders pivotal to its success. For the first time, R\&I and evidence-based practice aims were explicit with the 2012-2017 nursing and midwifery strategic document. This was further defined clearer measurable outcomes in the updated N\&M strategic plan (2017-2021) and supported the Trust's ongoing alignment with international standards of excellence in nursing care such as American Nurses Credentialing Center (2019). Since 2011, the workforce of N\&M with doctoral level preparation has grown from one to sixteen.

University Hospital Southampton NHS Foundation Trust (UHSFT) is a tertiary centre in the Wessex Region, and one of the largest acute providers in the south of England. It provides local inpatient services for a population of 1.9 million, with a tertiary service for adult services for 4 million, and children's services for 5.6 million. Local services are predominantly provided to Southampton City (SCCCG) and West Hampshire CCG (WHCCG). Regional work comes from the rest of Hampshire, Sussex, Surrey, Wiltshire, the Isle of Wight, and Dorset. For some more specialist services, patients travel from Devon, 
Cornwall, the Channel Islands, Berkshire, Oxfordshire, Buckinghamshire and Northamptonshire. The Trust plays an important role in teaching and training health professionals of the future, and undertakes research and development, in close partnership with the University of Southampton. Further NIHRfunded infrastructure is hosted by University Hospital Southampton including: a Biomedical Research Centre (BRC) specialising in respiratory, critical care and nutrition research, the Wessex Applied Research Collaboration (ARC), Wessex Clinical Research Network (CRN), and the South Central Research Design Service, and the university hosts the Southampton Clinical Trials Unit (CTU).

NMAHP capacity building started in earnest over a decade ago with a joint professorial appointment in 2009, with investment from both the Trust and the university. This provided leadership for the building of capacity and capability of aspiring NMAHP clinical academics. In 2011 the Trust published a Nursing and Allied Health Professions clinical research strategy that sought to ensure this group of health professionals had the means to make a full contribution to the production of leading-edge clinical research that could impact on care provision and improve outcomes (Latter et al., 2009; Westwood et al., 2018). The strategy outlined specific goals related to developing ('Our people, our partnerships and our organisation') and provided the focus to develop a UHSFT clinical doctoral research fellowship programme. The collaboration between the Trust and the UHSFT fostered a supportive environment for developing a Clinical Academic Careers structure for NMAHPs. This was cemented with the establishment of the Southampton Academy of Research (SoAR) in 2016, which provided a centralised pan-professional training hub and main point of contact for health professionals, research scientists and clinical research staff. Support for NMAHPs in particular has led to an increase in the number of successful NIHR PCAF applications from $5 \%$ to $14 \%$ of the total number awarded by this scheme to UHSFT in 2019.

\section{Structures and strategies to engage, enthuse and empower clinical academics}

Clinical academic training should not be viewed as a pursuit to a purely academic career and the development of such integrated roles within NHS organisations require effective structural empowerment (Coombs et al., 2012; Strickland 2017). Examples of structural empowerment and strategies are outlined below, with a focus predominantly on foundational 'pre-doctorate' activities at NUH, followed by a greater focus on post-doctorate by UHSFT.

\section{Foundation pre-doctoral structures}

In 2011, a benefits realisation exercise and engagement with frontline staff at NUH revealed that significant work was needed to embed foundational structures to promote research and evidence-based practice, and to enthuse and empower individuals even to consider themselves capable of undertaking clinical academic training and careers.

Supplemented by the findings from a Florence Nightingale Foundation (FNF) travel scholarship, a clinically driven Evidence in Practice programme was developed aiming to encourage those curious about CACs but lacking confidence to develop their skills and undertake a local quality improvement project (Fitzsimons \& Cooper, 2012). Currently in its seventh year of delivery this aligns with an organisational commitment to embedding Shared Governance leadership (Dinning et al., 2014) leadership across the N\&M workforce.

During this time, early engagement with senior divisional leaders across NUH resulted in a number of staff at different levels securing travel scholarships, sources including the Winston Churchill Foundation, the FNF and an in-house bespoke scholarship funded by the Queens Medical Centre (QMC) League of Friends. In addition, the creation of innovative foundational roles such as the Chief Nurse Excellence in Care Junior Fellowship (Bramley et al., 2018) provided additional opportunities for staff to undertake early career exposure to the clinical academic landscape. 
Celebration and showcasing of these successes, and alignment of CAC outcomes within the N\&M and Trust strategy, enabled individuals to access the CAC training pathway with support from transformational divisional leaders. Staff successfully secured places to study on the NIHR-funded masters in research at the University of Nottingham and Coventry University, in addition to regional HEE-funded internships at all levels of the pathway.

Individuals gain $\mathrm{PhD}$ funding from a variety of others sources including the Collaboration for Leadership in Applied Health Research and Care, East Midlands (EM-CLAHRC), Economic Social Research Council (ESRC), Wellbeing of Women, and University studentships. While some of these opportunities provided full funding for staff, others needed additional matched funding, requiring creative negotiation and combinations of clinical roles alongside $\mathrm{PhD}$ studentship study, a model that has provided a blueprint for clinical academic training roles to become embedded in clinical practice from the outset. This model of $\mathrm{PhD}$ funding combined with clinical practice has been successful in promoting N\&M CACs at NUH, with 16 post-doctoral and 10 in doctoral training in 2019. On reflection, this drive and focus on regional opportunities, although positive, has resulted in limited focus on national programmes. This is a focus oo further development in order to secure a balanced portfolio of maximising all funding opportunities.

In 2017, the launch of the Institute of Nursing and Midwifery Care Excellence (https://www.nuh.nhs.uk/ins) has utilised existing funding and income generation to create opportunities for project/research assistants at pre- and post-doctoral levels. This aims to build, not only individual capacity and capability, but also the visibility, profile, and contribution of clinical academic skills within and beyond primary research. As a critical mass of clinical academics has developed, this has enabled the utilisation of skills across all clinical divisions, with a growing number of partners across the region. Being able to 'pay this success forward' is an important part of the future at NUH. As a result of this, the East Midlands Clinical Academic Practitioner Network was formed in 2017 in collaboration with EMCLAHRC, and has been instrumental in providing regional support that has positively impacted the CAC agenda at other NHS Trusts and supported successful NIHR applications across the pathway. Further income from HEE Midlands and East has resulted in a current program of outreach support within the IMPACCT initiative.

\section{Post-doctoral structures and development}

The UHSFT research and development strategy from 2017 onwards included the desire to 'establish a cohesive and consistent 'core' offer for medical and non-medical trainees to enable career development, education and training of the next generation of world class translational research leaders' (UHSFT, 2017, p. 14). This in effect required the establishment of a talent management protocol.

One of the ways that UHSFT has achieved success for NMAHPs is through ring-fenced funds for transitional fellowships to enable those who are post-doctoral to carve out time for the development of high-quality grant and fellowship applications and submission of publications. SoAR has also facilitated post-doctoral researchers to apply for funding for interns to work alongside this group of emerging independent researchers. This serves two purposes: it enables the lead researcher to advance their research portfolio, and supervision and mentorship skills, whilst those new to research can develop their knowledge and skills, and experience the life of a clinical academic (Westwood et al., 2018).

SoAR also provides coaching and mentorship for doctoral and post-doctoral researchers and tracks their progress along the clinical academic pathway. Keeping a close eye on individuals' careers makes it easier to signal relevant funding and training opportunities. One of the key actions required to sustain postdoctoral positions within the Trust is to develop joint appointments between Trust and university. These posts are hard to develop, require commitment from both parties and can take more than twelve months to plan and establish, so negotiation needs to start early. SoAR has developed template job descriptions, available to other Trusts, that could make these posts easier to establish. Committed talent management fits with the NIHR Academy's resolve to monitor post-award support for NIHR personal fellowship awardees. Organisations seen not to meet commitments made to fellowship holders may in the future be challenged by the NIHR. In response, a joint piece of work has begun between the Trust and university to 
establish a policy about arrangement for fellowships, including the process of agreeing and subsequently meeting commitments during and following a fellowship.

On reflection, there remains a gap in the current offer. Whilst the clinical academic pathway identifies and supports specific pre-doctoral, doctoral and post-doctoral training, less attention is paid currently to other aspects of pursuit of a CAC, in particular, how to manage the competing demands of research delivery, publication, and further grant application, alongside a successful clinical career and also gaining an understanding of how to build a research group. Organisations must focus on this at an early stage if they are to become genuinely committed to developing a pool of clinical academic talent, so that junior researchers understand all aspects of what is required to pursue a successful CAC. In addition, equal attention must be given to achieving clinical and leadership-related skills and knowledge, along with the development of positions that employ skills that espouse improvements in patient care, for example through quality improvement activities.

Finally, when post-doctoral staff aspire to securing a joint appointment between the university and NHS organisation they can face challenges in managing what may seem to be competing priorities. The NHS organisation is focused on ensuring high-quality clinical service from the individual, whilst the university will often require a contribution to undergraduate and post-graduate education as well as setting the objectives of securing research income, managing current research projects and ensuring impact through publications and other activities. Managing these different streams of work can be difficult, especially when also trying to achieve an effective work/life balance. To help people achieve this there is the need for robust joint approaches to appraisal and job planning; in addition, attention must be paid to ensuring that individuals develop resilience and that they can effectively negotiate and manage competing demands of the different organisations.

\section{Realising benefits for staff and patient experience}

When successfully supported and enabled, NMAHP clinical academics provide substantial benefits to organisations, not only in providing a positive career development opportunity through driving portfolios of research with impact on patient care and services, but as leaders embedded in clinical services, with a transferable skill-set to support service transformation and development across Integrated Care Systems (ICS). Clinical academics are highly valued in both Nottingham and Southampton for their contribution to three key dimensions of impact set out in the following sections.

\section{Improving patient outcomes - developing services and interventions}

In Nottingham, significant strides are being made in understanding and improving the experience of children and young people with mental health conditions and their families under the leadership of a postdoctoral NIHR Clinical Lecturer and jointly appointed professor (Carter et al., 2019; Manning et al., 2018). Similarly, the care and experiences of adults and children with Chronic Lymphedema are being transformed under the leadership of an internationally renowned Clinical Professor and local PhD clinical academics and specialist teams (Quéré et al., 2019), with strategic ambitions to transform services at NUH and across the wider community of Nottinghamshire.

Not all service development at NUH has been driven by clinical academics with such extensive experience. Those at the beginning of the clinical academic training journey have been instrumental in transforming the care and outcomes for patients. A recipient of an ICA internship at pre-doctoral level has been standardising the assessment process of mental health patients who attend the Emergency Department. This has resulted in reduced lengths of stay and greater staff satisfaction for patients in acute mental health crisis. Other aspiring clinical academics, in their early career, have examined evidence in relation to the transition of care (Wells and Manning, 2017) and the improvement of care for patients with dementia in the recovery room (Edis, 2017) as part of their Chief Nurse Excellence in Care Junior Fellowship.

Staff at UHSFT, at different stages of the clinical academic pathway, evidence equally impressive results and potential. Working collaboratively on the care of patients awaiting major surgery, a pre-doctoral 
nurse and professorial-level Respiratory Physiologist are developing a prehabilitation and perioperative medicine service (PPOM). Early results suggest improved outcomes for these patients, resulting in longterm behaviour change.

A cancer nurse, just completing her doctorate in the field of social networks and cancer survivorship, secured a role that involved the adoption of holistic needs assessment by clinical teams. This role is leading and evaluating an approach to managing patients with cancer with co-morbidity across the primary and secondary interface, and is offering post-treatment nurse-led survivorship care clinics in her speciality of lymphoma (Wagland et al., 2015).

One post-doctoral Allied Health Professional has explored and identified the facilitators and barriers to meeting older adults' health literacy needs in clinical practice, focusing on patients with cancer. This has provided greater understanding of these needs, informing further work to improve communication with potential for reduced morbidity/mortality and increased patients' satisfaction and self-management abilities (Brooks et al., 2017).

\section{Developing evidence-based policies and procedures}

Collaborations fostered by NMAHP clinical academics in the NUH INMCE have also seen benefits when seeking to develop the evidence base. A recent collaboration with the Trent Simulation Centre has led to a team of post-doctoral clinical academic nurses and midwives developing a conceptual framework for the use of in-situ simulation in clinical practice. This project has led to further research that is needed to strengthen the evidence base in this area and is set to be utilised by HEE in developing their national strategy to ensure equity of access to simulation education across England.

UHSFT's Associate Professor and Clinical Nurse Specialist in infection prevention has looked at portable bladder ultrasound scanning technology, a fast, painless and non-invasive way to measure urine volume in the bladder (Westwood et al., 2013). As an alternative to bladder catheterisation, it has been shown to reduce urinary tract infection (UTI) significantly while increasing patient satisfaction. She undertook an economic assessment to establish a strategy for future use. The amount of money saved by the Trust in using the scanner and avoiding catheterisation was estimated to be around $£ 1$.23million per year, not including the cost of bacteraemia attributed to urinary tract infections. Associated reduced expenditure by avoiding the need to use a scanner, such as fewer treatment delays and overnight stays in hospital, were recognised as additional savings.

\section{Leadership of transformation and effectiveness effort}

As trailblazers within their specialties, NUH NMAHPs are often transformational leaders within their fields of clinical practice. At NUH, this leadership is seen at all levels across the CAC pathway from band 5 upwards and extends to NHS and NUH strategic priority areas. Examples of this can be seen across the ICS within maternity transformation, where three NUH midwives, two of whom are post-doctoral while one is undertaking doctoral training, are working closely with ICS colleagues to implement work streams to realise the Better Births vision (NHS England, 2017). N\&M leadership capability has also been a key feature of the Trust's ambition to achieve exemplary patient outcomes as part of its journey to care excellence (ANCC Magnet).

One individual at UHSFT has developed a service and care pathway, as part of a post-doctoral fellowship from the NIHR in her joint role of lecturer and advanced nurse practitioner, that enables a person-centred approach and supports shared decision-making in relation to renal dialysis and end of life care (Murphy et al., 2017).

In addition, a physiotherapy professor provides a good example of how clinical academics can develop a joint appointment that delivers on clinical, academic and research priorities. The focus of her academic and clinical work is promoting musculoskeletal health, and her research portfolio includes:

communication and decision-making; improving patient experience; improving the health of patients with Parkinson's (through dance); and promoting independence using web-based technologies for people with 
back pain (Carr, 2015). She is a keen mentor and coach of less experienced clinical academics, and works with the NIHR Academy to develop the next generation of clinical academics.

These examples show that investing at an individual level in NMAHPs' clinical academic careers at all levels of the pathway has had an impact across the wider organisation. This investment has also led to the retention of a significant number of NMAHPs within both organisations who have been supported to pursue CACs. Organisational strategic ambitions to support this agenda has been fundamental in creating a community of individuals who are forming a critical mass of leaders, with the transferable skills to realise benefits for both patients and staff.

\section{Conclusion}

Clinical academic roles for NMAHP professions offer significant positive gains for patient and staff outcomes in NHS organisations, examples of which are showcased here. This is a complex challenge, however, requiring a complex solution, involving a range of activities and support. Pivotal factors include sustained strategic commitment, structures that enable the training, and role development of aspiring and current clinical academics. When crafted and created, this has the potential to develop an innovative workforce that feels valued and developed, with the right knowledge and skills to provide the highest quality care for a contemporary NHS healthcare system.

\section{Acknowledgements}

Kay Mitchell is a National Institute for Health Research (NIHR) Senior Nurse and Midwife Research Leader. The views expressed in this article are those of the authors and not necessarily those of the NIHR, or the Department of Health and Social Care.

Professor Alison Richardson is a National Institute for Health Research (NIHR) Senior Investigator. The views expressed in this article are those of the author(s) and not necessarily those of the NHS, the NIHR, or the Department of Health.

\section{ORCID}

Joanne Cooper:

Kay Mitchell:

Alison Richardson:

Louise Bramley:

\author{
https://orcid.org/0000-0003-1340-3872 \\ https://orcid.org/0000-0001-6393-8475 \\ https://orcid.org/0000-0003-3127-5755 \\ https://orcid.org/0000-0002-0425-1734
}

\section{References}

American Nurses Credentialing Center. (2019). Magnet model. https://www.nursingworld.org/organizational-programs/magnet/magnet-model/.

Bramley, L., Manning, J. C., \& Cooper, J. (2018). Engaging and developing front-line clinical nurses to drive care excellence: Evaluating the Chief Nurse Excellence in Care Junior Fellowship initiative. Journal of Research in Nursing, 23(8), 678-689. https://doi.org/10.1177/1744987118808843

Brooks, C., Ballinger, C., Nutbeam, G. D., \& Adams, J. (2017). The importance of building trust and tailoring interactions when meeting older adults' health literacy needs. Disability and Rehabilitation, 39(23), 2428-2435. https://doi.org/10.1080/09638288.2016.1231849

Carr, C. (2015). Clinical Notes: A music therapist's investigation into the benefits of her therapy is just one example of the new directions research is taking on the ground. Health Service Journal, 18(Suppl), 8. 
Carter, T., Walker, G. M., Aubeeluck, A., \& Manning, J. C. (2019). Assessment tools of immediate risk of self-harm and suicide in children and young people: A scoping review. Journal of Child Health Care, 23(2), 178-199. https://doi.org/10.1177/1367493518787925

Coombs, M., Latter, S., \& Richardson, A. (2012). Developing a clinical academic career pathway for nursing. British Journal of Nursing, 21(18), 1084-1086, 1088-1090. https://doi.org/10.12968/bjon.2012.21.18.1084

Dinning, A., Cooper, J., Taylor K., Damas, R., \& Hailes, L. (2014). 'Knowing why we do what we do' Establishing a unit practice council to improve evidence based nursing practice in acute medicine using appreciative inquiry. FoNS Improvement Insights, 10(5), 1.

Edis, H. (2017). Improving care for patients with dementia in the recovery room. British Journal of Nursing, 26(20), 1102-1108. https://doi.org/10.12968/bjon.2017.26.20.1102

Fitzsimons, E., \& Cooper, J. (2012). Embedding a culture of evidence-based practice. Nursing Management, 19(7), 14-19. https://doi.org/10.7748/nm2012.11.19.7.14.c9370

Jonker, L., \& Fisher, S. J. (2018). The correlation between National Health Service trusts' clinical trial activity and both mortality rates and care quality commission ratings: A retrospective crosssectional study. Public Health, 157, 1-6. https://doi.org/10.1016/i.puhe.2017.12.022

Jonker, L., Fisher, S. J., \& Dagnan, D. (2019). Patients admitted to more research-active hospitals have more confidence in staff and are better informed about their condition and medication: Results from a retrospective cross-sectional study. Journal of Evaluation in Clinical Practice. https://doi.org/10.1111/jep.13118

Latter, S., Clark, J. M., Geddes, C., \& Kitsell, F. (2009). Implementing a clinical academic career pathway in nursing; Criteria for success and challenges ahead. Journal of Research in Nursing, 14(2), 137-148. https://doi.org/10.1111/jep.13118

Manning, J. C., Walker, G. M., Carter, T., Aubeeluck, A., Witchell, M., \& Coad, J. (2018). Children and Young People-Mental Health Safety Assessment Tool (CYP-MH SAT) study: Protocol for the development and psychometric evaluation of an assessment tool to identify immediate risk of self-harm and suicide in children and young people (10-19 years) in acute paediatric hospital settings. BMJ Open, 8(4), e020964. https://doi.org/10.1136/bmjopen-2017-020964

Murphy, E., Germain, M. J., \& Murtagh, F. (2017). Palliative nephrology: Time for new insights. American Journal of Kidney Diseases, 70(5), 593-595. https://doi.org/10.1053/j.ajkd.2017.07.008

NHS England. (2017). Implementing Better Births: A resource pack for Local Maternity Systems. https://www.england.nhs.uk/wp-content/uploads/2017/03/nhs-guidance-maternity-servicesv1.pdf

Quéré, I., Palmier, S., Nøerregaard, S., Pastor, J., Sykorova, M., Dring, E., Franks, P. J., Murray, S., Keeley, V., Bermark, S., Karlsmark, T., Kyne, N., Colgan, M.-P., Coulombe, M.-M., Mestre, S., Mercier, G., \& Moffatt, C. J. (2019). LIMPRINT: Estimation of the prevalence of lymphoedema/chronic oedema in acute hospital in in-patients. Lymphatic Research and Biology, 17(2), 135-140. https://doi.org/10.1089//rb.2019.0024

Strickland, K. (2017). Developing an infrastructure to support clinical academic careers. British Journal of Nursing, 26(22), 1249-1252. https://doi.org/10.12968/bjon.2017.26.22.1249 
University Hospital Southampton, NHS Foundation Trust (UHSFT). (2017). Research for all University Hospital Southampton NHS Foundation Trust Research Strategy https://www.uhs.nhs.uk/Media/Southampton-Clinical-Research/RD-strategy-2017-2022.pdf

Wagland, R., Fenlon, D., Tarrant, R., Howard-Jones, G., \& Richardson, A. (2015). Rebuilding selfconfidence after cancer: A feasibility study of life-coaching. Support Care in Cancer, 23(3), 651-659. https://doi.org/10.1007/s00520-014-2399-5

Wells, F., \& Manning, J. (2017). Transition of care from children's to adult services. Nursing Children and Young People, 29(8), 30-34. https://doi.org/10.7748/ncyp.2017.e897

Westwood, G., Fader, M., Roberts, L., Green, S. M., Prieto, J., \& Bayliss-Pratt, L. (2013). How clinical academics are transforming patient care. Health Service Journal, 123(6368), 28-29.

Westwood, G., Richardson, A., Latter, S., Macleod Clark, J., \& Fader, M. (2018). Building clinical academic leadership capacity: Sustainability through partnership. Journal of Research in Nursing, 23(4), 346-357. https://doi.org/10.1177/1744987117748348 